\title{
Relationships of the Mediterranean dietary pattern with insulin resistance and diabetes incidence in the Multi-Ethnic Study of Atherosclerosis (MESA)
}

\author{
Eunice E. Abiemo ${ }^{1}$, Alvaro Alonso ${ }^{1}$, Jennifer A. Nettleton ${ }^{2}$, Lyn M. Steffen ${ }^{1}$, Alain G. Bertoni ${ }^{3}$, \\ Aditya Jain ${ }^{4}$ and Pamela L. Lutsey ${ }^{1 *}$ \\ ${ }^{1}$ Division of Epidemiology and Community Health, School of Public Health, University of Minnesota, 1300 S. \\ 2nd Street, Suite 300, Minneapolis, MN 55454, USA \\ ${ }^{2}$ Division of Epidemiology and Disease Control, University of Texas Health Science Center, Houston, TX, USA \\ ${ }^{3}$ Divisions of Public Health Sciences and Internal Medicine, Wake Forest University, Winston-Salem, NC, USA \\ ${ }^{4}$ Department of Radiology, Johns Hopkins University, Baltimore, MD, USA
}

(Submitted 25 March 2012 - Final revision received 20 June 2012 - Accepted 25 June 2012 - First published online 30 August 2012)

\begin{abstract}
Type 2 diabetes (T2D) is a highly prevalent but preventable disorder. We assessed the association between an a priori Mediterranean diet (MeDiet) score and fasting glucose and insulin at baseline and incident T2D after a 6-year follow-up in the Multi-Ethnic Study of Atherosclerosis. Dietary intake was measured at baseline using a 127-item FFQ in 5390 men and women aged 45-84 years free of prevalent diabetes and clinical CVD. A MeDiet score was created based on the intake of ten food components: vegetables; whole grains; nuts; legumes; fruits; ratio of monounsaturated:saturated fat; red and processed meat; dairy products; fish; alcohol. Multivariable linear and proportional hazards models were used to estimate the association of the MeDiet, categorised in quintiles, with baseline insulin and glucose, and incident diabetes, respectively. The models were adjusted for demographic, physiological and behavioural characteristics. After multivariable adjustment, individuals with a higher MeDiet score had lower baseline mean insulin levels (Q1: 5·8 (95\% CI 5.6, 6.0) $\mu$ mol/1; Q5: $4.8(95 \% \mathrm{CI} 4 \cdot 6,5 \cdot 0) \mu \mathrm{mol} / \mathrm{l} ; P$ for trend $<0 \cdot 0001)$. A higher MeDiet score was also associated with significantly lower glucose levels after basic adjustment, but was attenuated after adjustment for waist circumference. During the follow-up, 412 incident diabetes events accrued. The MeDiet was not significantly related to the risk of incident diabetes ( $P$ for trend $=0.64$ ). In summary, greater consistency with a Mediterranean-style diet, reflected by a higher a priori MeDiet score, was cross-sectionally associated with lower insulin levels among non-diabetics, and with lower blood glucose before adjustment for obesity, but not with a lower incidence of diabetes.
\end{abstract}

Key words: Mediterranean diet: Insulin: Glucose: Diabetes: Multi-Ethnic Study of Atherosclerosis (MESA)

Diabetes affects approximately twenty-four million people in the USA, $8 \%$ of the population, and is currently the seventh leading cause of death. It has been projected that the prevalence of diabetes will reach $26 \%$ by $2050^{(1)}$. Accounting for about $95 \%$ of all diagnosed cases in the USA, type 2 diabetes (T2D) results in complications such as kidney failure, amputations and blindness, affecting the quality of life ${ }^{(2)}$. Management of the disease also poses a huge medical burden and economic impact, making it a current public health priority. T2D can, however, be prevented through healthy diets, other lifestyle modifications such as weight loss and the use of medication ${ }^{(3)}$.

The Mediterranean diet (MeDiet) is the traditional diet of people living in olive-growing regions bordering the Mediterranean Sea. It is of public health interest due to the observation that adults living in these areas have historically had one of the lowest incidences of chronic diseases in the world and one of the highest life expectancies ${ }^{(4)}$. This diet is characterised by a high consumption of whole grains, olive oil, legumes, vegetables, fruits and cereals, moderate to high consumption of fish and moderate to low consumption of meat and meat products and milk and dairy products. Alcohol in the form of wine is often consumed at meals ${ }^{(5)}$. Extensive research has demonstrated a beneficial effect of specific dietary components of the MeDiet on weight loss, normalising insulin resistance, and the risk of developing T2D and $\mathrm{CVD}^{(5-7)}$. The MeDiet is widely viewed as 'health promoting', both among the scientific community and among the general public. Possible mechanisms by which intake of the MeDiet may be associated with lower diabetes risk include fibre increasing satiety through prolonged mastication and antioxidants reducing the stress of $\beta$-cell dysfunction and insulin

Abbreviations: IFG, impaired fasting glucose; MeDiet, Mediterranean diet; MESA, Multi-Ethnic Study of Atherosclerosis; T2D, type 2 diabetes. 
resistance $^{(8)}$. Relationships of the MeDiet to disease risk may also be mediated through the anti-inflammatory effects of vitamins, minerals, antioxidants and unsaturated fat - particularly olive oil - present in high levels in the MeDiet ${ }^{(5,7,9,10)}$.

There is limited evidence of the association between insulin resistance - as a precursor of diabetes - and the MeDiet in non-diabetic individuals. Additionally, little research has investigated whether racial/ethnic heterogeneity exists in the relationship between the intake of a Mediterranean-style diet and the incidence of diabetes and the onset of insulin resistance. We hypothesised that in a community-based sample free of diabetes and CVD, a high conformity to the Mediterranean-style diet would be associated cross-sectionally with lower insulin resistance and prospectively with a reduced risk of $\mathrm{T} 2 \mathrm{D}$ incidence. Our MeDiet index was created a priori, and focused on those food groups commonly attributed to the Mediterranean cuisine, i.e. vegetables, whole grains, nuts, legumes, fruits, MUFA:SFA ratio and fish.

\section{Methods}

\section{Study population}

The Multi-Ethnic Study of Atherosclerosis (MESA) is a prospective population-based cohort study of 6814 persons aged 45-84 years who self-identified as Hispanic, non-Hispanic Caucasian, African-American and Asian Chinese ${ }^{(11)}$. The study was initiated in July 2000 to determine the characteristics associated with the prevalence and progression of subclinical CVD to clinically overt CVD, as well as to investigate demographic differences and identify risk factors for CVD incidence. Baseline information was collected from enrollees, all of whom were free from clinical CVD, at six US field centres: Chicago, IL; Los Angeles County, CA; New York, NY; Forsyth County, NC; St Paul, MN and Baltimore, MD. A detailed study protocol and inclusion criteria can be found at www.mesa-nhlbi.org. The present study was conducted according to the guidelines laid down in the Declaration of Helsinki, and all procedures involving human subjects were approved by the Institutional Review Boards from each study centre. All participants gave written informed consent.

\section{Dietary pattern assessment}

At baseline, a 127-item FFQ was used to assess usual dietary intake of participants over the past year. For each questionnaire item, participants were asked to report their frequency of consumption of various foods from among nine categories, ranging from rarely or never to two or more servings/d (six or more servings/d for beverages), and also their serving size as either small, medium or large. Servings per $\mathrm{d}$ were calculated from these categories. The questionnaire was patterned after the FFQ used in the Insulin Resistance Atherosclerosis Study, which has been validated in nonHispanic white, African-American and Hispanic persons ${ }^{(12)}$.

To ascertain conformity to a Mediterranean-style diet, a ten-point a priori alternate MeDiet score was created. The MeDiet score was adapted to the US population from a scoring system modelled for Greek populations and focuses on higher consumption of plant foods, monounsaturated fat, fish and lower intake of animal products and saturated fat $^{(6,13)}$. As detailed in Table 1 , the score included ten food components: vegetables (excluding potatoes); whole grains; nuts; legumes; fruits; ratio of monounsaturated:saturated fat; red and processed meat; whole-fat dairy products; fish; alcohol $^{(14)}$. Participants with intakes above the median intake of traditional foods in the MeDiet (i.e. vegetables, whole grains, nuts, legumes, fruits, MUFA:SFA ratio, fish) received 1 point, while those below the median received 0 points. For potentially detrimental foods inversely associated with the MeDiet (i.e. red/processed meats, whole-fat dairy products), those with intakes below the median received 1 point; otherwise, they received 0 points. For example, red or processed meat below the median intake received 1 point. Alcohol intake received 1 point if consumed in moderate amounts $(5-15 \mathrm{~g} / \mathrm{d})$ and 0 otherwise $(<5$ and $>15 \mathrm{~g} / \mathrm{d})$. The points were then summed. The final MeDiet score ranged from 0 to 10 , with a higher score indicating a closer resemblance to the MeDiet.

\section{Outcome ascertainment}

Insulin resistance was cross-sectionally characterised using mean baseline fasting glucose and fasting serum insulin

Table 1. Food group components of the ten-point Mediterranean diet score: the Multi-Ethnic Study of Atherosclerosis (2000-2)

\begin{tabular}{|c|c|c|}
\hline Food group & Foods included & Criteria for 1 point ${ }^{*}$ \\
\hline Vegetables & $\begin{array}{l}\text { All vegetables (cruciferous vegetables, dark yellow } \\
\text { vegetables, green leafy vegetables, other } \\
\text { vegetables) except potatoes }\end{array}$ & Greater than median intake \\
\hline Legumes & Legumes, soya & Greater than median intake \\
\hline Fruit & Fruits, fruit juice, avocado, tomato & Greater than median intake \\
\hline Nuts & Seeds, nuts & Greater than median intake \\
\hline Whole grains & All whole-grain products & Greater than median intake \\
\hline Fish & All fish & Greater than median intake \\
\hline Red meat & Red and processed meats & Less than median intake \\
\hline $\begin{array}{l}\text { Ratio of monounsaturated: } \\
\text { saturated fat }\end{array}$ & - & Greater than median intake \\
\hline Alcohol & Total alcohol (beer, liquor, wine) & Moderate intake $(>5$ and $<15 \mathrm{~g} / \mathrm{d})$ \\
\hline Dairy products & Whole milk, high-fat cheeses and sauces & Less than median intake \\
\hline
\end{tabular}

* 0 points if these criteria are not met. 
levels among non-diabetics. Fasting serum glucose was measured at each examination using the thin-film adaptation of the glucose oxidase method on the Vitros analyser (Johnson \& Johnson Clinical Diagnostics, Inc.). To ensure consistency of the fasting serum glucose assay over the examinations, 200 samples from each of the four examinations were reanalysed over a short time period to recalibrate the original observations. Fasting serum insulin levels were determined by a RIA method using the Linco Human Insulin-Specific RIA Kit (Linco Research, Inc.). These assays were conducted at the Collaborative Studies Clinical Laboratory at Fairview University Medical Center ${ }^{(15-17)}$.

Table 2. Baseline characteristics* by quintiles of the Mediterranean dietary score: the Multi-Ethnic Study of Atherosclerosis (2000-2) (Mean values and standard deviations; number of participants and percentages)

\begin{tabular}{|c|c|c|c|c|c|c|c|c|c|c|}
\hline Quintile†... & \multicolumn{2}{|c|}{1} & \multicolumn{2}{|c|}{2} & \multicolumn{2}{|c|}{3} & \multicolumn{2}{|c|}{4} & \multicolumn{2}{|c|}{5} \\
\hline MeDiet score range... & \multicolumn{2}{|c|}{$0-3$} & \multicolumn{2}{|c|}{4} & \multicolumn{2}{|c|}{5} & \multicolumn{2}{|c|}{6} & \multicolumn{2}{|c|}{$7-10$} \\
\hline \multirow[t]{2}{*}{$n .}$. & \multicolumn{2}{|c|}{1240} & \multicolumn{2}{|c|}{967} & \multicolumn{2}{|c|}{1051} & \multicolumn{2}{|c|}{932} & \multicolumn{2}{|c|}{1200} \\
\hline & $n$ & $\%$ & $n$ & $\%$ & $n$ & $\%$ & $n$ & $\%$ & $n$ & $\%$ \\
\hline \multicolumn{11}{|l|}{ Demographics } \\
\hline Age (years) & & & & & & & & & & \\
\hline Mean & & & & & & & & & & \\
\hline SD & & & & & & & & & & \\
\hline Women & 676 & 54.5 & 522 & $54 \cdot 0$ & 552 & 52.5 & 505 & $54 \cdot 2$ & 629 & $52 \cdot 4$ \\
\hline Racial/ethnic group & & & & & & & & & & \\
\hline White & 547 & $44 \cdot 1$ & 396 & $41 \cdot 0$ & 425 & $40 \cdot 4$ & 377 & $40 \cdot 5$ & 558 & $46 \cdot 5$ \\
\hline Chinese & 68 & 5.5 & 99 & $10 \cdot 2$ & 163 & $15 \cdot 5$ & 165 & $17 \cdot 7$ & 180 & $15 \cdot 0$ \\
\hline African-American & 293 & $23 \cdot 6$ & 243 & $25 \cdot 1$ & 244 & $23 \cdot 2$ & 230 & $24 \cdot 7$ & 305 & $25 \cdot 4$ \\
\hline Hispanic & 332 & $26 \cdot 8$ & 229 & $23 \cdot 7$ & 219 & $20 \cdot 8$ & 160 & $17 \cdot 2$ & 157 & $13 \cdot 1$ \\
\hline Formal education & & & & & & & & & & \\
\hline Less than high school diploma & 249 & $20 \cdot 2$ & 193 & $20 \cdot 0$ & 182 & $17 \cdot 3$ & 132 & $14 \cdot 2$ & 122 & $10 \cdot 2$ \\
\hline High school or some college & 628 & $50 \cdot 8$ & 484 & $50 \cdot 1$ & 464 & $44 \cdot 2$ & 132 & $44 \cdot 7$ & 471 & $39 \cdot 3$ \\
\hline College diploma & 359 & $29 \cdot 0$ & 289 & $29 \cdot 9$ & 405 & 38.5 & 132 & $41 \cdot 1$ & 606 & $50 \cdot 5$ \\
\hline Gross family income & & & & & & & & & & \\
\hline$<\$ 20000$ & 277 & $23 \cdot 0$ & 222 & $24 \cdot 1$ & 249 & 24.5 & 194 & $21 \cdot 4$ & 213 & $18 \cdot 3$ \\
\hline$\$ 20000-<\$ 50000$ & 460 & $38 \cdot 2$ & 379 & $41 \cdot 1$ & 338 & $33 \cdot 2$ & 324 & $35 \cdot 8$ & 360 & 30.9 \\
\hline$\geq \$ 50000$ & 466 & $38 \cdot 8$ & 322 & 34.9 & 431 & $42 \cdot 3$ & 388 & $42 \cdot 8$ & 592 & $50 \cdot 8$ \\
\hline $\mathrm{BMI}\left(\mathrm{kg} / \mathrm{m}^{2}\right)$ & & & & & & & & & & \\
\hline Mean & & & & & & & & & & \\
\hline SD & & & & & & & & & & \\
\hline Waist circumference $(\mathrm{cm})$ & & & & & & & & & & \\
\hline Mean & & & & & & & & & & \\
\hline SD & & & & & & & & & & \\
\hline Behavioural & & & & & & & & & & \\
\hline Current smoking & 236 & $19 \cdot 1$ & 136 & $14 \cdot 1$ & 122 & $11 \cdot 6$ & 83 & $8 \cdot 8$ & 90 & $7 \cdot 5$ \\
\hline Pack-years of cigarette smoking & & & & & & & & & & \\
\hline Mean & & & & & & & & & & \\
\hline $\mathrm{SD}$ & & & & & & & & & & \\
\hline Physical activity (MET-min/week) & & & & & & & & & & \\
\hline Mean & & & & & & & & & & \\
\hline SD & & & & & & & & & & \\
\hline Physiological & & & & & & & & & & \\
\hline SBP $(\mathrm{mmHg})$ & & & & & & & & & & \\
\hline Mean & & & & & & & & & & \\
\hline SD & & & & & & & & & & \\
\hline $\mathrm{DBP}(\mathrm{mmHg})$ & & & & & & & & & & \\
\hline Mean & & & & & & & & & & \\
\hline SD & & & & & & & & & & \\
\hline TAG (mg/l) & & & & & & & & & & \\
\hline Mean & & & & & & & & & & \\
\hline SD & & & & & & & & & & \\
\hline LDL-cholesterol (mg/l) & & & & & & & & & & \\
\hline Mean & & & & & & & & & & \\
\hline SD & & & & & & & & & & \\
\hline HDL-cholesterol (mg/l) & & & & & & & & & & \\
\hline Mean & & & & & & & & & & \\
\hline SD & & & & & & & & & & \\
\hline Hypertensive medication use & 318 & $25 \cdot 6$ & 293 & $30 \cdot 3$ & 324 & $30 \cdot 8$ & 292 & $31 \cdot 3$ & 365 & $30 \cdot 4$ \\
\hline Statins use & 139 & $11 \cdot 2$ & 133 & $13 \cdot 7$ & 142 & 13.5 & 122 & $13 \cdot 1$ & 179 & 14.9 \\
\hline
\end{tabular}

MeDiet, Mediterranean diet; MET, metabolic equivalent; systolic blood pressure; DBP, diastolic blood pressure.

* Participants with prevalent diabetes were excluded.

†Higher quintile represents closer conformity to the MeDiet. 
To determine incident diabetes, we excluded from the analyses individuals with diabetes at baseline, defined as fasting blood glucose $\geq 7.0 \mathrm{mmol} / 1(1260 \mathrm{mg} / \mathrm{l})$, self-reported diabetes or using hypoglycaemic drugs. During follow-up examinations $2(2002-3), 3(2004-5)$ and $4(2005-7)$, participants without diabetes at baseline who met any of the above three criteria were considered to have incident T2D. Person-years were accrued from baseline until the date of the examination at which incident diabetes was identified, loss to follow-up or the date of examination 4 .

\section{Covariate assessment}

Demographic data were obtained during the baseline examination (2000-2) with a standardised questionnaire and calibrated devices. Participants self-reported their racial/ ethnic groups and were characterised as Caucasian, Chinese, African-American and Hispanic. The annual gross family income was categorised as $<\$ 20000, \$ 20000-<\$ 50000$ and $\geq \$ 50000$, and the level of formal education was classified as less than high school diploma, high school or some college and college diploma. Height was measured with a stadiometer with a level bubble (Accu-Hite Measuring device; Seca GmbH \& Company KG) and weight with a Detecto platform balance scale (Titus Home Health Care). BMI was calculated as weight (in $\mathrm{kg}$ ) divided by the square of height (in $\mathrm{m}^{2}$ ). Waist circumference (in $\mathrm{cm}$ ) was measured at the level of the umbilicus. Resting seated blood pressure was measured three times using a Dinamap model Pro 100 automated oscillometric sphygmomanometer (Critikon), and the average of the last two measurements was used in the analysis.

Additional variables such as use of hypertensive medication, use of statins (3-hydroxy-3-methyl-glutaryl (HMG)-CoA reductase inhibitors), cigarette smoking status and time spent in moderate to vigorous exercise (metabolic equivalent (MET)-min/week) were obtained from a combination of selfadministered and interviewer-administered questionnaires. Pack-years of smoking, defined as number of years smoking times packs per $d$ (cigarettes per $d$ divided by 20), were then calculated $^{(15,18-20)}$.

\section{Statistical analyses}

All analyses were performed using SAS software (version 9.2; SAS Institute). Of the 6814 MESA participants, we excluded those with prevalent diabetes ( $n$ 859), missing values ( $n$ 24) and people with unrealistic dietary intake ( $n$ 541). Unrealistic dietary intake was defined as energy intake of $<2092$ or $>20920 \mathrm{~kJ} / \mathrm{d}(<500$ or $>5000 \mathrm{kcal} / \mathrm{d})$. The present final analytic sample included 5390 individuals.

Cross-sectional demographic, behavioural and physiological characteristics across quintiles of the MeDiet score were quantified using means and proportions. We used multivariable linear regression to assess the association of the MeDiet with insulin resistance. Separate analyses were conducted for fasting glucose and insulin at baseline across MeDiet quintiles. After checking for normality of these outcomes, serum insulin was found to be skewed and was therefore log-transformed for multivariate analyses. The adjusted mean values were then back-transformed to obtain geometric means. Adjusted means at each MeDiet quintile were obtained by entering the MeDiet quintiles into the models as indicator variables. To test the linear trend across the quintiles of the MeDiet score, quintiles were entered into the models as continuous variables.

We used three multivariable models in the present analysis. The first model (model 1) adjusted for age, sex, race/ethnicity and study site. Model 2 adjusted for model 1 variables plus educational level, family income, physical activity, smoking status and total energy intake. Further, to assess the effect of body adiposity and how this might mediate the association of the MeDiet and the outcomes, waist circumference was included in the final model (model 3). In sensitivity analyses, we explored substituting BMI for waist circumference.

Cox proportional hazards regression models were used to estimate the hazard ratios of developing T2D by MeDiet quintiles. The adjustment approach was similar to the crosssectional analysis, with quintile 1 as the reference for the Cox regression model. We further tested whether there were interactions by sex and race/ethnicity in the relationship between the MeDiet and the outcomes by including crossproduct terms in our models. In sensitivity analyses, we evaluated the risk of incident impaired fasting glucose (IFG) or diabetes in a subset free of IFG and diabetes at baseline.

\section{Results}

The 5390 participants in the present analytic sample were on average 62 (SD 10) years old and 54\% were female. The racial/ethnic distribution was as follows: $43 \%$ Caucasian; 13\% Chinese; 24\% African-American; 20\% Hispanic. The average MeDiet score was 5.0 (SD 1.9) on a 0-10-point scale within the study population. Participants who had higher MeDiet scores, indicating a high conformity to the MeDiet, were more likely to be female, more educated, have higher incomes, a smaller waist circumference and be non-smokers (Table 2). In this population of non-diabetics, the mean glucose level was $895 \mathrm{mg} / \mathrm{l}$, and the geometric mean insulin level was $5 \cdot 25 \mu \mathrm{mol} / \mathrm{l}$. Furthermore, $15 \cdot 7 \%$ ( $n$ 841) of the analytic sample had IFG, as defined by fasting glucose levels of $1000-1250 \mathrm{mg} / \mathrm{l}$.

Participants with a higher MeDiet score had lower insulin levels after model 2 adjustments (Q1: 5.8 (95\% CI 5.6, 6.0) $\mu \mathrm{mol} / \mathrm{l}$; Q5: 4.8 (95\% CI 4.6, 5.0) $\mu \mathrm{mol} / \mathrm{l} ; P$ for trend $<0.0001$; Table 3 ). The results were similar to model 1 , and this relationship remained significant in model 3 , which was further adjusted for waist circumference $(P$ for trend $<0.0001)$. Upon stratification by sex, the association among men remained significant across all models, while among women, the association was attenuated after adjustment for waist circumference (model $3, P$ for trend=0.11). After model 2 adjustments, mean fasting glucose was also lower for individuals in MeDiet quintile 5 (890 (95\% CI 884 , 896) $\mathrm{mg} / \mathrm{l})$ relative to those in MedDiet quintile 1 (903 (95\% CI 897,909$) \mathrm{mg} / \mathrm{l})(P$ for trend=0.009). The relationship, 
Table 3. Glucose and insulin levels by the Mediterranean dietary score quintiles: the Multi-Ethnic Study of Atherosclerosis (2000-2) (Adjusted mean values and $95 \%$ confidence intervals)

\begin{tabular}{|c|c|c|c|c|c|c|c|c|c|c|c|c|}
\hline \multirow[b]{2}{*}{ Quintile* } & \multicolumn{2}{|c|}{1} & \multicolumn{2}{|c|}{2} & \multicolumn{2}{|c|}{3} & \multicolumn{2}{|c|}{4} & \multicolumn{2}{|c|}{5} & \multirow{2}{*}{$\begin{array}{c}\beta \text { for one } \\
\text { quintile increase }\end{array}$} & \multirow{2}{*}{$\begin{array}{l}P \text { for } \\
\text { trend }\end{array}$} \\
\hline & Mean & $95 \% \mathrm{Cl}$ & Mean & $95 \% \mathrm{Cl}$ & Mean & $95 \% \mathrm{Cl}$ & Mean & $95 \% \mathrm{Cl}$ & Mean & $95 \% \mathrm{Cl}$ & & \\
\hline \multicolumn{13}{|c|}{ Total population } \\
\hline \multicolumn{13}{|c|}{ Glucose (mg/l) } \\
\hline Model $1 \dagger$ & 903 & 898,909 & 891 & 885,897 & 896 & 890,902 & 892 & 886,899 & 890 & 884,896 & -0.27 & 0.005 \\
\hline Model $2 \ddagger$ & 903 & 897,909 & 889 & 882,895 & 896 & 890,902 & 892 & 885,898 & 890 & 884,896 & -0.26 & 0.009 \\
\hline Model 3§ & 899 & 893,904 & 889 & 883,895 & 895 & 889,901 & 893 & 887,900 & 894 & 888,899 & -0.07 & 0.450 \\
\hline \multicolumn{13}{|c|}{ Insulin $(\mu \mathrm{mol} / \mathrm{I}) \|$} \\
\hline Model 1† & $5 \cdot 7$ & $5 \cdot 5,5 \cdot 9$ & $5 \cdot 3$ & $5 \cdot 1,5 \cdot 5$ & $5 \cdot 3$ & $5 \cdot 1,5 \cdot 5$ & $5 \cdot 1$ & $4 \cdot 9,5 \cdot 3$ & $4 \cdot 8$ & $4 \cdot 7,5 \cdot 0$ & -0.04 & $<0.0001$ \\
\hline Model $2 \ddagger$ & $5 \cdot 8$ & $5 \cdot 6,6 \cdot 0$ & $5 \cdot 3$ & $5 \cdot 1,5 \cdot 5$ & $5 \cdot 3$ & $5 \cdot 1,5 \cdot 5$ & $5 \cdot 0$ & $4 \cdot 8,5 \cdot 2$ & $4 \cdot 8$ & $4 \cdot 6,5 \cdot 0$ & -0.04 & $<0.0001$ \\
\hline Model 3§ & $5 \cdot 5$ & $5 \cdot 4,5 \cdot 7$ & $5 \cdot 3$ & $5 \cdot 1,5 \cdot 5$ & $5 \cdot 3$ & $5 \cdot 1,5 \cdot 4$ & $5 \cdot 1$ & $4 \cdot 9,5 \cdot 3$ & $5 \cdot 0$ & $4 \cdot 9,5 \cdot 2$ & -0.02 & $<0.0001$ \\
\hline \multicolumn{13}{|l|}{ Males } \\
\hline \multicolumn{13}{|c|}{ Glucose (mg/l) } \\
\hline Model $1 \dagger$ & 927 & 918,935 & 910 & 900,919 & 911 & 902,920 & 911 & 901,921 & 906 & 897,914 & -0.42 & 0.003 \\
\hline Model $2 \ddagger$ & 927 & 918,936 & 907 & 898,917 & 913 & 904,922 & 911 & 901,920 & 905 & 896,913 & -0.43 & 0.004 \\
\hline Model 3§ & 922 & 914,931 & 907 & 898,916 & 913 & 904,922 & 913 & 903,922 & 908 & 899,916 & -0.26 & 0.070 \\
\hline \multicolumn{13}{|c|}{ Insulin $(\mu \mathrm{mol} / \mathrm{l})||$} \\
\hline Model 1† & $6 \cdot 1$ & $5 \cdot 8,6 \cdot 4$ & $5 \cdot 3$ & $5 \cdot 0,5 \cdot 6$ & $5 \cdot 2$ & $4 \cdot 9,5 \cdot 5$ & 4.9 & $4 \cdot 7,5 \cdot 2$ & $5 \cdot 0$ & $4 \cdot 7,5 \cdot 2$ & -0.05 & $<0.0001$ \\
\hline Model 2‡ & $6 \cdot 2$ & $5 \cdot 9,6 \cdot 5$ & $5 \cdot 3$ & $5 \cdot 0,5 \cdot 7$ & $5 \cdot 3$ & $5 \cdot 0,5 \cdot 6$ & 4.9 & $4 \cdot 6,5 \cdot 2$ & 4.9 & $4 \cdot 6,5 \cdot 1$ & -0.06 & $<0.0001$ \\
\hline Model 3§ & $5 \cdot 8$ & $5 \cdot 6,6 \cdot 1$ & $5 \cdot 3$ & $5 \cdot 1,5 \cdot 6$ & $5 \cdot 3$ & $5 \cdot 0,5 \cdot 5$ & $5 \cdot 0$ & $4 \cdot 8,5 \cdot 3$ & $5 \cdot 1$ & $4 \cdot 9,5 \cdot 3$ & -0.03 & $<0.0001$ \\
\hline \multicolumn{13}{|l|}{ Females } \\
\hline \multicolumn{13}{|c|}{ Glucose (mg/l) } \\
\hline Model 1† & 884 & 876,891 & 875 & 866,883 & 883 & 875,892 & 876 & 867,885 & 877 & 869,885 & -0.13 & 0.32 \\
\hline Model $2 \ddagger$ & 883 & 875,891 & 872 & 863,881 & 881 & 873,890 & 875 & 866,884 & 877 & 869,885 & -0.10 & 0.48 \\
\hline Model 3§ & 878 & 871,886 & 873 & 865,882 & 880 & 872,888 & 876 & 867,885 & 881 & 874,889 & 0.09 & 0.50 \\
\hline \multicolumn{13}{|c|}{ Insulin $(\mu \mathrm{mol} / \mathrm{I})||$} \\
\hline Model 1† & $5 \cdot 4$ & $52,5 \cdot 7$ & $5 \cdot 3$ & $5 \cdot 0,5 \cdot 6$ & 5.4 & $5 \cdot 1,5 \cdot 6$ & $5 \cdot 2$ & $4 \cdot 9,5 \cdot 5$ & $4 \cdot 7$ & $4 \cdot 5,5 \cdot 0$ & -0.03 & 0.0002 \\
\hline Model $2 \ddagger$ & 5.4 & $5 \cdot 2,5 \cdot 7$ & $5 \cdot 2$ & $5 \cdot 0,5 \cdot 5$ & $5 \cdot 3$ & $5 \cdot 1,5 \cdot 6$ & $5 \cdot 2$ & $4 \cdot 9,5 \cdot 4$ & 4.8 & $4 \cdot 6,5 \cdot 0$ & -0.03 & 0.0005 \\
\hline Model $3 \S$ & $5 \cdot 2$ & $5 \cdot 0,5 \cdot 4$ & $5 \cdot 3$ & $5 \cdot 1,5 \cdot 5$ & $5 \cdot 3$ & $5 \cdot 0,5 \cdot 5$ & $5 \cdot 2$ & $5 \cdot 0,5 \cdot 4$ & $5 \cdot 0$ & $4 \cdot 8,5 \cdot 2$ & -0.01 & 0.11 \\
\hline
\end{tabular}

*Higher quintile represents closer conformity to the Mediterranean diet.

Model 1 adjusted for age, sex, race/ethnicity and study site.

¥ Model 2 adjusted for model 1 + educational level, family income, smoking status, physical activity and total energy intake.

$\S$ Model 3 adjusted for model $2+$ waist circumference.

Values for quintiles $1-5$ are geometric means. $\beta$ for one quintile increase in the Mediterranean diet score are presented on the log scale. The log-scale $\beta$ multiplied by 100 gives the percentage change per one quintile increase in the Mediterranean diet score. For example, using model 1 and the total population, each one quintile increase in the Mediterranean diet score is associated with a $4 \%$ lower insulin level. 
Table 4. Hazard ratios (HR) of type 2 diabetes across quintiles of the Mediterranean dietary score: the Multi-Ethnic Study of Atherosclerosis (2000-7) (Hazard ratios and $95 \%$ confidence intervals)

\begin{tabular}{|c|c|c|c|c|c|c|c|c|c|c|c|c|}
\hline \multirow[t]{2}{*}{ Quintiles*... } & \multirow{2}{*}{$\begin{array}{c}1 \\
\mathrm{HR}\end{array}$} & \multicolumn{2}{|c|}{2} & \multicolumn{2}{|c|}{3} & \multicolumn{2}{|c|}{4} & \multicolumn{2}{|c|}{5} & \multicolumn{2}{|c|}{$\begin{array}{l}\text { HR for one } \\
\text { quintile increase }\end{array}$} & \multirow{2}{*}{$\begin{array}{l}P \text { for } \\
\text { trend }\end{array}$} \\
\hline & & $\mathrm{HR}$ & $95 \% \mathrm{Cl}$ & $\mathrm{HR}$ & $95 \% \mathrm{Cl}$ & $\mathrm{HR}$ & $95 \% \mathrm{Cl}$ & $\mathrm{HR}$ & $95 \% \mathrm{Cl}$ & $\mathrm{HR}$ & $95 \% \mathrm{Cl}$ & \\
\hline \multicolumn{13}{|l|}{ Total population } \\
\hline Events $(n)$ & 99 & \multicolumn{2}{|c|}{73} & \multirow{2}{*}{\multicolumn{2}{|c|}{83}} & \multirow{2}{*}{\multicolumn{2}{|c|}{$\begin{array}{c}68 \\
3702\end{array}$}} & \multicolumn{2}{|c|}{89} & & & \\
\hline Person-years & 4936 & \multirow{2}{*}{\multicolumn{2}{|c|}{$\begin{array}{c}3886 \\
18.8\end{array}$}} & \multirow{2}{*}{\multicolumn{2}{|c|}{$\begin{array}{l}4230 \\
19.6\end{array}$}} & & & \multirow{2}{*}{\multicolumn{2}{|c|}{5013}} & & & \\
\hline Incidence rate $†$ & $20 \cdot 1$ & & & & & \multicolumn{2}{|c|}{$18 \cdot 4$} & & & & & \\
\hline Model 1f & 1 (reference) & 0.93 & $0.69,1.26$ & 1.00 & $0.75,1.35$ & 0.96 & $0.70,1.31$ & 0.93 & $0.69,1.25$ & 0.99 & $0.92,1.06$ & 0.71 \\
\hline Model $2 \S$ & 1 (reference) & 0.88 & $0.65,1.20$ & 0.97 & $0.72,1.31$ & 0.93 & $0.67,1.28$ & 0.91 & $0.67,1.23$ & 0.98 & $0.92,1.06$ & 0.64 \\
\hline Model 3\| & 1 (reference) & 1.01 & $0.74,1.38$ & 1.04 & $0.77,1.41$ & 1.08 & $0.78,1.50$ & 1.09 & $0.80,1.49$ & 1.02 & $0.95,1.10$ & 0.51 \\
\hline \multicolumn{13}{|l|}{ Males } \\
\hline Events $(n)$ & 41 & \multicolumn{2}{|c|}{40} & \multicolumn{2}{|c|}{41} & \multicolumn{2}{|c|}{35} & \multicolumn{2}{|c|}{42} & & & \\
\hline Person-years & 2281 & \multirow{2}{*}{\multicolumn{2}{|c|}{$\begin{array}{l}1799 \\
22.2\end{array}$}} & \multirow{2}{*}{\multicolumn{2}{|c|}{$\begin{array}{l}2048 \\
20 \cdot 0\end{array}$}} & \multirow{2}{*}{\multicolumn{2}{|c|}{$\begin{array}{l}1691 \\
20.7\end{array}$}} & \multirow{2}{*}{\multicolumn{2}{|c|}{$\begin{array}{l}2350 \\
17.9\end{array}$}} & & & \\
\hline Incidence rate & 18.0 & & & & & & & & & & & \\
\hline Model $1 \ddagger$ & 1 (reference) & 1.19 & $0.77,1.84$ & 1.09 & $0.70,1.69$ & 1.19 & $0.75,1.87$ & 1.02 & $0.65,1.58$ & 1.00 & $0.91,1.10$ & 0.98 \\
\hline Model $2 \S$ & 1 (reference) & $1 \cdot 12$ & $0.71,1.75$ & 1.07 & $0.68,1.66$ & 1.08 & $0.67,1.73$ & 0.95 & $0.60,1.50$ & 0.99 & $0.89,1.09$ & 0.79 \\
\hline Model 3\| & 1 (reference) & 1.24 & $0.79,1.94$ & 1.15 & $0.74,1.81$ & 1.26 & $0.78,2.04$ & $1 \cdot 11$ & $0.70,1.76$ & 1.02 & $0.92,1.13$ & 0.69 \\
\hline \multicolumn{13}{|l|}{ Females } \\
\hline Events $(n)$ & 58 & \multicolumn{2}{|c|}{33} & \multicolumn{2}{|c|}{42} & \multicolumn{2}{|c|}{33} & \multicolumn{2}{|c|}{47} & & & \\
\hline Person-years & 2656 & & 887 & & 82 & & 011 & & 64 & & & \\
\hline Incidence rate $†$ & $21 \cdot 8$ & & 5.8 & & 9.2 & & 6.4 & & 7.6 & & & \\
\hline Model $1 \ddagger$ & 1 (reference) & 0.73 & $0.48,1.12$ & 0.93 & $0.62,1.39$ & 0.77 & $0.50,1.18$ & 0.86 & $0.58,1.27$ & 0.97 & $0.88,1.06$ & 0.51 \\
\hline Model $2 \S$ & 1 (reference) & 0.70 & $0.45,1.09$ & 0.88 & $0.58,1.34$ & 0.78 & $0.50,1.22$ & 0.89 & $0.59,1.35$ & 0.98 & $0.89,1.08$ & 0.70 \\
\hline Model 3\| & 1 (reference) & 0.82 & $0.53,1.29$ & 0.95 & $0.62,1.45$ & 0.92 & $0.59,1.45$ & 1.12 & $0.74,1.71$ & 1.03 & $0.93,1.14$ & 0.55 \\
\hline
\end{tabular}

${ }^{*}$ Higher quintile represents closer conformity to the Mediterranean diet.

Incidence per 1000 person-years.

the 1 adjusted for age, sex, race/ethnicity and study site.

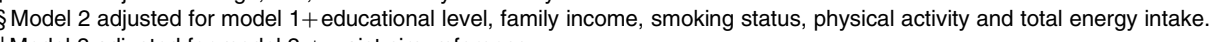

|| Model 3 adjusted for model 2 + waist circumference. 
however, disappeared after adjusting for waist circumference (model 3, $P$ for trend $=0.45$ ).

After 6.6 years of follow-up, 412 participants (7.6\%) developed T2D. In this study population, the MeDiet was not significantly related to the risk of T2D incidence, and this relationship was consistent across sex (Table 4) and racial/ethnic groups (results not shown for the racial/ethnic groups). The model 2 multivariable hazard ratio of T2D among those in the highest quintile of the MeDiet score $v$. the lowest quintile was 0.91 (95\% CI $0.67,1 \cdot 23, P$ for trend=0.64; Table 4).

In additional analyses, there were no significant interactions by race/ethnicity or sex in the relationship between the MeDiet and insulin levels, glucose levels or the incidence of diabetes. The results were similar when we adjusted for BMI instead of waist circumference (data not shown). Furthermore, we also evaluated the risk of incident IFG or diabetes in a subset free of IFG and diabetes at baseline. There was no evidence that the MeDiet score was associated with a lower risk of incident IFG or diabetes. The hazard ratio for one quintile change in the MeDiet score was 0.98 (95\% CI 0.94, 1.02).

\section{Discussion}

In the present population-based, multi-ethnic sample, a higher consumption of the Mediterranean-style diet was cross-sectionally associated with lower blood glucose and insulin levels, before adjustment for adiposity. Adjustment for waist circumference attenuated the association between the MeDiet and blood glucose; however, the relationship between the MeDiet and insulin levels remained statistically significant. The MeDiet was not related to the incidence of T2D.

Similar to the present findings, an inverse cross-sectional association was found between indices of glucose homeostasis and adherence to the MeDiet among non-diabetic subjects in Greece ${ }^{(21)}$. A recent study in French households comparing computer-simulated personalised diets with a $7 \mathrm{~d}$ food record of adults from French households in meeting dietary recommendations has also shown that foods typical of the MeDiet such as unrefined grains, legumes, nuts, fruits, fish and vegetables were efficient ways to achieve overall nutrient adequacy $^{(9)}$. Additionally, current evidence indicates that adherence to a Mediterranean dietary pattern together with maintenance of ideal body weight appears to be an excellent strategy to reduce T2D risk ${ }^{(10,22)}$.

Contrary to the present hypothesis, the Mediterranean dietary pattern was not related to a lower T2D incidence in this population. This is in contrast to previous published findings, such as the PREDIMED (Preverción con Dieta Mediterránea) study, a randomised trial in Spain, which indicated that consumption of the MeDiet led to a $50 \%$ reduction in diabetes incidence over 4 years among non-diabetics at high CVD risk, and the observational Nurses' Health Study which found that after a 20-year follow-up, consumption of a Mediterranean-style diet was associated with a significantly lower risk of CVD, CHD and stroke ${ }^{(6,23)}$. A recent large prospective study (European Prospective Investigation Into Cancer and Nutrition Study: The InterAct project), which used nine dietary characteristic components of the MeDiet (score range $0-18$ ), has also shown that adherence to the MeDiet was associated with a small reduction in the risk of developing $\mathrm{T}_{2} \mathrm{D}^{(24)}$

There are several limitations of the present study, which may explain the lack of association in the MESA population between the MedDiet score and incident diabetes. First, the MeDiet score might be inadequate for a multi-ethnic population in the USA, with dietary patterns very different from those traditionally observed in Mediterranean countries. As a second limitation, the present study attempted to define a Mediterranean dietary pattern from usual diets of participants using a dietary assessment tool not specifically designed to measure conformity to the MeDiet. Not all of the distinct food components of the traditional MeDiet were included in the questionnaire (e.g. olive oil). Furthermore, many of the components were part of line items, which included nonMeDiet foods in addition to the MeDiet food items. Our attempt to tease out specific food components from composite or mixed dishes might have possibly led to either overestimation or underestimation of some food categories. Third, measurement error associated with changes in the diet over the duration of follow-up could cause misclassification of the exposure. Unlike the Nurses' Health Study where there was updated dietary information, the MESA had only one dietary measure. This might be a possible explanation for the differences in the present findings ${ }^{(6)}$. Lastly, if the association between the MeDiet and incident diabetes is small in magnitude, we may have been underpowered to detect a relationship. Despite these limitations, the present study also has several important strengths, including the diverse population, use of objectively identified diabetes (not only self-report), and the highly standardised serum processing, anthropometric measurements and covariate assessment across the study centres.

Notably, a previous analysis of the MESA sample identified two empirically derived dietary patterns associated with the risk of diabetes ${ }^{(17)}$. In contrast to the previous work, our MeDiet index focused a priori on only those food groups that were commonly attributed to the Mediterranean cuisine. However, the percentage agreement of quintile ranks for the MeDiet score and each of the previous dietary patterns were about $35 \%$, indicating a fair to moderate agreement in how the dietary patterns were associated with the risk of T2D.

In the present study, we found that a high consumption of the MeDiet was associated with significantly lower serum insulin levels, but after adjustment for obesity was not related to glucose levels or the incidence of T2D. The new dietary guidelines for Americans, which are based on current epidemiological evidence, reiterate the need for a more plant-based diet, less processed meat, and more low-fat dairy products and seafood, which are typical components of the Mediterranean dietary pattern. The synergistic effect of these individual components of the MeDiet may give this dietary pattern its numerous beneficial properties in mitigating chronic diseases such as T2D. Overall, the present study supports the existing evidence that consistent consumption of a Mediterranean-type diet may lead to reduced risk for T2D. Given the beneficial properties of the traditional MeDiet, further research should determine whether this pattern 
is applicable to and leads to similar health benefits in diverse ethnic groups with other culinary traditions.

\section{Acknowledgements}

This study was supported by contracts N01-HC-95159 to N01-HC-95169 from the National Heart, Lung, and Blood Institute. Dr Nettleton was supported by grant 5K01DK082729-02 from the National Institute of Diabetes and Digestive and Kidney Diseases. The authors thank the other investigators, the staff and the participants of the MESA study for their valuable contributions. A full list of participating MESA investigators and institutions can be found at http://www.mesa-nhlbi.org. E. E. A. and A. A. developed the research question and operationalised the MeDiet score. E. E. A. and P. L. L. conducted the data analysis and led in the writing of the manuscript. E. E. A., A. A., J. A. N., L. M. S., A. G. B. and P. L. L. made substantial conceptual contributions and revisions. The authors do not have any conflict of interest.

\section{References}

1. Boyle JP, Thompson TJ, Gregg EW, et al. (2010) Projection of the year 2050 burden of diabetes in the US adult population: dynamic modeling of incidence, mortality, and prediabetes prevalence. Popul Health Metr 8, 29.

2. American Diabetes Association (2011) Diagnosis and classification of diabetes mellitus. Diabetes Care 34, S62-S69.

3. Knowler WC, Barrett-Connor E, Fowler SE, et al. (2002) Reduction in the incidence of type 2 diabetes with lifestyle intervention or metformin. $N$ Engl J Med 346, 393-403.

4. Keys A, Menotti A, Karvonen MJ, et al. (1986) The diet and 15-year death rate in the Seven Countries Study. Am J Epidemiol 124, 903-915.

5. Estruch R, Martínez-González MA, Corella D, et al. (2006) Effects of a Mediterranean-style diet on cardiovascular risk factors: a randomized trial. Ann Intern Med 145, 1-11.

6. Fung TT, Rexrode KM, Mantzoros CS, et al. (2009) Mediterranean diet and incidence of and mortality from coronary heart disease and stroke in women. Circulation 119, 1093-1100.

7. Martínez-González MA, de la Fuente-Arrillaga C, NúñezCórdoba JM, et al. (2008) Adherence to Mediterranean diet and risk of developing diabetes: prospective cohort study. BMJ 336, 1348-1351.

8. Ben-Avraham S, Harman-Boehm I, Schwarzfuchs D, et al. (2009) Dietary strategies for patients with type 2 diabetes in the era of multi-approaches: review and results from the Dietary Intervention Randomized Controlled Trial (DIRECT). Diabetes Res Clin Pract 86, S41-S48.

9. Maillot M, Issa C, Vieux F, et al. (2011) The shortest way to reach nutritional goals is to adopt Mediterranean food choices: evidence from computer-generated personalized diets. Am J Clin Nutr 94, 1127-1137.
10. Kastorini CM \& Panagiotakos DB (2010) Mediterranean diet and diabetes prevention: myth or fact? World J Diabetes $\mathbf{1}$, 65-67.

11. Bild DE, Bluemke DA, Burke GL, et al. (2002) Multi-ethnic study of atherosclerosis: objectives and design. $A m J$ Epidemiol 156, 871-881.

12. Mayer-Davis EJ, Vitolins MZ, Carmichael SL, et al. (1999) Validity and reproducibility of a food frequency interview in a Multi-Cultural Epidemiology Study. Ann Epidemiol 9, $314-324$

13. Trichopoulou A, Costacou T, Barnia C, et al. (2003) Adherence to a Mediterranean diet and survival in a Greek population. N Engl J Med 348, 2599-2608.

14. Nettleton JA, Steffen LM, Mayer-Davis EJ, et al. (2006) Dietary patterns are associated with biochemical markers of inflammation and endothelial activation in the Multi-Ethnic Study of Atherosclerosis (MESA). Am J Clin Nutr 83, 1369-1379.

15. Bertoni AG, Burke GL, Owusu JA, et al. (2010) Inflammation and the incidence of type 2 diabetes: the Multi-Ethnic Study of Atherosclerosis (MESA). Diabetes Care 33, 804-810.

16. Lutsey PL, Jacobs DR Jr, Kori S, et al. (2007) Whole grain intake and its cross-sectional association with obesity, insulin resistance, inflammation, diabetes and subclinical CVD: the MESA Study. Br J Nutr 98, 397-405.

17. Nettleton JA, Steffen LM, Ni H, et al. (2008) Dietary patterns and risk of incident type 2 diabetes in the Multi-Ethnic Study of Atherosclerosis (MESA). Diabetes Care 31, 1777-1782.

18. Lutsey PL, Pereira MA, Bertoni AG, et al. (2010) Interactions between race/ethnicity and anthropometry in risk of incident diabetes: the Multi-Ethnic Study of Atherosclerosis. Am J Epidemiol 172, 197-204.

19. Nettleton JA, Lutsey PL, Wang Y, et al. (2009) Diet soda intake and risk of incident metabolic syndrome and type 2 diabetes in the Multi-Ethnic Study of Atherosclerosis (MESA). Diabetes Care 32, 688-694.

20. Kandula NR, Diez-Roux AV, Chan C, et al. (2008) Association of acculturation levels and prevalence of diabetes in the Multi-Ethnic Study of Atherosclerosis (MESA). Diabetes Care 31, 1621-1628.

21. Panagiotakos DB, Tzima N, Pitsavos C, et al. (2007) The association between adherence to the Mediterranean diet and fasting indices of glucose homeostasis: the ATTICA study. J Am Coll Nutr 26, 32-38

22. Salas-Salvadó J, Martínez-González MA, Bulló M, et al. (2011) The role of diet in the prevention of type 2 diabetes. Nutr Metab Cardiovasc Dis 21, B32-B48.

23. Salas-Salvadó J, Bulló M, Babio N, et al. (2011) Reduction in the incidence of type 2 diabetes with the Mediterranean diet: results of the PREDIMED-Reus nutrition intervention randomized trial. Diabetes Care 34, 14-19.

24. InterAct Consortium (2011) Mediterranean diet and type 2 diabetes risk in the European Prospective Investigation into Cancer and Nutrition (EPIC) study: the InterAct project. Diabetes Care 34, 1913-1918. 